\title{
A microbial survey of the International Space Station (ISS)
}

\author{
Jenna M Lang ${ }^{\text {Corresp., }}{ }^{1}$ ， David A Coil ${ }^{1}$ ， Russell Y Neches ${ }^{1}$ ， Wendy E Brown ${ }^{1,2}$ ， Darlene Cavalier ${ }^{2,3,4}$, Mark \\ Severance $^{2,4}$ ， Jarrad T Hampton-Marcell ${ }^{5,6}$, Jack A Gilbert ${ }^{7,8}$, Jonathan A Eisen ${ }^{1,9,10}$ \\ ${ }^{1}$ Genome Center, University of California, Davis, Davis, California, United States \\ 2 Science Cheerleader \\ 3 The Consortium for Science, Policy \& Outcomes, Arizona State University, Tempe, Arizona, United States \\ 4 Scistarter.org \\ 5 Biosciences Division, Argonne National Laboratory, Lemont, Illinois, United States \\ 6 Department of Biological Sciences, University of Illinois at Chicago, Chicago, Illinois, United States \\ 7 University of Chicago, Argonne National Laboratory, Lemont, Illinois, United States \\ 8 Institute for Genomics and Systems Biology, Argonne National Laboratory, Lemont, Illinois, United States \\ 9 Evolution and Ecology, University of California, Davis, Davis, California, United States \\ 10 Medical Microbiology and Immunology, University of California, Davis, Davis, CA, United States \\ Corresponding Author: Jenna M Lang \\ Email address: jennomics@gmail.com
}

Background: Modern advances in sequencing technology have enabled the census of microbial members of many natural ecosystems. Recently, attention is increasingly being paid to the microbial residents of human-made, built ecosystems, both private (homes) and public (subways, office buildings, and hospitals). Here, we report results of the characterization of the microbial ecology of a singular built environment, the International Space Station (ISS). This ISS sampling involved the collection and microbial analysis (via 16S rDNA PCR) of 15 surfaces sampled by swabs onboard the ISS. This sampling was a component of Project MERCCURI (Microbial Ecology Research Combining Citizen and University Researchers on ISS). Learning more about the microbial inhabitants of the "buildings" in which we travel through space will take on increasing importance, as plans for human exploration continue, with the possibility of colonization of other planets and moons.

Results: Sterile swabs were used to sample 15 surfaces onboard the ISS. The sites sampled were designed to be analogous to samples collected for 1) the Wildlife of Our Homes project and 2) a study of cell phones and shoes that were concurrently being collected for another component of Project MERCCURI. Sequencing of the 16S rDNA genes amplified from DNA extracted from each swab was used to produce a census of the microbes present on each surface sampled. We compared the microbes found on the ISS swabs to those from both homes on Earth and data from the Human Microbiome Project.

Conclusions: While significantly different from homes on Earth and the Human Microbiome Project samples analyzed here, the microbial community composition on the ISS was more similar to home surfaces than to the human microbiome samples. The ISS surfaces are species-rich with 1036-4294 operational taxonomic units (OTUs per sample). There was no discernible biogeography of microbes on the 15 ISS surfaces, although this may be a reflection of the small sample size we were able to obtain. 


\section{A microbial survey of the International Space Station (ISS)}

2 Jenna M. Lang (jennomics@gmail.com) Genome Center, University of California, Davis, 3 CA, USA

4 David A. Coil (coil.david@gmail.com). Genome Center, University of California, Davis, 5 CA, USA

6 Russell Y. Neches (russell@vort.org). Genome Center, University of California, Davis, 7 CA, USA

8 Wendy E. Brown (thewendybrown@gmail.com). Science Cheerleader, Genome Center,

9 University of California, Davis, CA, USA

10 Darlene Cavalier (darcav1@gmail.com). Science Cheerleader, SciStarter.org

11 Mark Severance (scicheerspaceguy@scistarter.org). Science Cheerleader, SciStarter.org

12 Jarrad Marcell (jhampton-marcell@anl.gov). Argonne National Laboratory, University of

13 Chicago, Lemont, IL, USA

14 Jack A. Gilbert (gilbert.jack@gmail.com). Argonne National Laboratory, University of

15 Chicago, Lemont, IL,

16 Jonathan A. Eisen (jaeisen@ucdavis.edu). Genome Center, Evolution and Ecology, 17 Medical Microbiology and Immunology, University of California, Davis, Davis, CA, 18 USA

\section{Abstract}

Background: Modern advances in sequencing technology have enabled the census of microbial members of many natural ecosystems. Recently, attention is increasingly being paid to the microbial residents of human-made, built ecosystems, both private (homes) and public (subways, office buildings, and hospitals). Here, we report results of the characterization of the microbial ecology of a singular built environment, the International Space Station (ISS). This ISS sampling involved the collection and microbial analysis (via 16S rDNA PCR) of 15 surfaces sampled by swabs onboard the ISS. This sampling was a component of Project MERCCURI (Microbial Ecology Research Combining Citizen and University Researchers on ISS). Learning more about the microbial inhabitants of the "buildings" in which we travel through space will take on increasing importance, as plans 
31 for human exploration continue, with the possibility of colonization of other planets and 32 moons.

33 Results: Sterile swabs were used to sample 15 surfaces onboard the ISS. The sites sampled were designed to be analogous to samples collected for 1) the Wildlife of Our Homes project and 2) a study of cell phones and shoes that were concurrently being collected for another component of Project MERCCURI. Sequencing of the 16S rDNA genes amplified from DNA extracted from each swab was used to produce a census of the microbes present on each surface sampled. We compared the microbes found on the ISS swabs to those from both homes on Earth and data from the Human Microbiome Project.

Conclusions: While significantly different from homes on Earth and the Human Microbiome Project samples analyzed here, the microbial community composition on the ISS was more similar to home surfaces than to the human microbiome samples. The ISS surfaces are species-rich with 1036-4294 operational taxonomic units (OTUs per sample). There was no discernible biogeography of microbes on the 15 ISS surfaces, although this may be a reflection of the small sample size we were able to obtain.

\section{Introduction}

There is a growing appreciation of the importance of microbial communities found in diverse environments from the oceans, to soil, to the insides and outsides of plants and animals. Recently, there has been an expanding focus on the microbial ecology of the "built environment" - human constructed entities like buildings, cars, and trains - places where humans spend a large fraction of their time. One relatively unexplored type of built environment is that found in space. As humans expand their reach into the solar system, with renewed interest in space travel, and with the possibility of the colonization of other planets and moons, it is of critical importance to understand the microbial ecology of the built environments being utilized for such endeavors.

Interest in the microbial occupants of spacecraft long precedes the launch of the International Space Station (ISS) (Trexler 1964)(Silverman 1971). Early work primarily focused on ensuring that the surfaces of spacecraft were free of microbial contaminants in an effort to avoid inadvertent panspermia (seeding other planets with microbes from Earth) (Pierson 2007). Work on human-occupied spacecraft such as Mir, Space Shuttles, and Skylab focused more on microbes with possible human health effects. With the launch of the ISS, it was understood that this new built environment would be permanently housing microbes as well as humans. Calls were made for a better understanding of microbial ecology and human-microbe interactions during extended stays in space (Pierson 2007) (Roberts, Garland, and Mills 2004) (Ott, Bruce, and Pierson 2004). Efforts were made to establish a baseline microbial census. For example, Novikova et al (Novikova et al. 2006) obtained more than 500 samples from the air, potable water, and surfaces of the ISS, over the course of 6 years.

These early studies were unavoidably limited by their reliance on culturing to identify microbial species. Culture-independent approaches were eventually implemented, 
71 including some small-scale 16S rDNA PCR surveys (Castro et al. 2004), and the Lab-On-a-

72 Chip Application Development Portable Test System (LOCAD-PTS) (Maule et al. 2009),

73 which allows astronauts to test surfaces for lipopolysaccharide (LPS - a marker for Gram

74 negative bacteria). Originally launched in 2006, the capability of the LOCAD-PTS was

75 expanded in 2009 to include an assay for fungi (beta-glucan, a fungal cell wall component)

76 and Gram positive bacteria (lipoteichoic acid, a component of the cell wall of Gram positive

77 bacteria.) The first large-scale, culture-independent 16S rDNA PCR survey was published

78 only in 2014 using the Roche 454 platform (pyrosequencing), looking at dust on the ISS

79 (Venkateswaran et al. 2014). A more recent study examined several samples collected on

80 the Japanese module of the ISS over a period of four years, also sequenced with

81 pyrosequencing (Ichijo et al. 2016). We report here on a further effort involving 16S rDNA

82 PCR and sequencing, using the Illumina platform, to examine the microbial communities

83 found on 15 surfaces inside the ISS. The advantage of Illumina sequencing, relative to

84 previous pyrosequencing efforts, is the significant increase in depth of sequencing. This

85 increased depth allowed us to analyze 15-20 times as many sequences as these earlier

86 studies.

87

88

89

90

91

92

93

94

95

96

97

98

99

100

101

102

103

104

105

106

107

108

The 15 surfaces sampled on the ISS were chosen by the Project MERCCURI team in an effort to make them analogous to 1 ) the surfaces sampled for the "Wildlife of Our Homes" project (http://homes.yourwildlife.org) (Dunn et al. 2013) (Barberán et al. 2015), which asked citizen scientists to swab nine surfaces in their homes, and 2) cell phone and shoe swab samples that were also being collected via Project MERCCURI. The sample matching is imperfect, for example doorsills were used in houses because they collect dust but in the microgravity of the ISS, dust accumulates in air filters. The motivation for choosing the sites in this way was both to increase public awareness of the microbiology of the built environment, as well as to begin to compare the microbial ecology of homes on Earth with the only current human home in space. We also present a comparison of the ISS swab results with data from 13 human body sites sampled via the Human Microbiome Project. This comparison was done to assess the potential human contribution to the microbial life on the ISS.

We have also compiled a collection of papers on space microbiology in an online resource to provide a more comprehensive historical perspective of this kind of work (see http://www.mendeley.com/groups/844031/microbiology-of-the-builtenvironment/papers/added/0/tag/space/).

\section{Methods}

\section{Surfaces swabbed:}

Astronauts were asked to swab 15 surfaces on the International Space Station. Below are their verbatim instructions. 
109 1. Audio Terminal Unit (telephone) hand held push-to-talk microphone located in the

110 forward portion of the US Lab Module

111 2. Audio Terminal Unit (telephone) hand held push-to-talk microphone located in the aft 112 portion of the US Lab Module

113 3. US Lab Robotic Work Station laptop PC keyboard used to control the robotic arm

114 4. US Lab Robotic Work Station hand controller used to control the movement of the 115 robotic arm

116 5. US Lab Robotic Work Station foothold, left side

117 6. US Lab Robotic Work Station foothold, right side

118 7. One of the main laptop keyboards in the US Lab used to control science experiments 119 and the systems of the space station

120 8. One of the vertical handrails on the equipment racks inside the US Lab

1219 . Air vent in the front portion of the US Lab

122 10. Air vent in the aft portion of the US Lab

123 11. Air vent located on the right crew sleep compartment

124 12. Tab used to open, close, and secure the Nomex privacy panel located on the starboard 125 crew sleep compartment

126 13. Air vent located on the port crew sleep compartment

127 14. Tab used to open, close, and secure the Nomex privacy panel located on the port crew 128 sleep compartment

129 15. Crew Choice Surface: Audio Terminal Unit (telephone) hand held push-to-talk

130 microphone located in the starboard portion of the Harmony module (Node 2).

131 Swabbing instructions as given to astronauts:

132 1. Setup Node-2 Camcorder to capture NanoRacks surface swab Ops throughout the US 133 LAB.

134 2. Retrieve a clean NanoRacks Swab Kit. Move to ISS location listed on NanoRacks Swab $135 \quad$ Kit label.

136 3. Remove cotton swab from NanoRacks Swab Kit, being careful not to touch the cotton 137 swab tip to avoid contamination.

138 4. Rub cotton swab vigorously against designated surface. Spin and turn the swab to 139 ensure maximum sample collection. 
140 5. Return cotton swab to NanoRacks Swab Kit and press to close (squeeze excess air out

141

142

143

144

145

146

147

148

149

150

151

152

153

154

155

156

157

158

159

160

161

162

163

164

165

166

167

168

169

170

171

172

173

174

175

176

177

178

179 of bag before sealing). Circle number of location swabbed and label with GMT (dd/hh:mm). If swab is contaminated by touching a surface other than the designated location on the label, Label NanoRacks Swab Kit with a large, "X" and move on to the next location. Notify POIC of NanoRacks Swab Kit S/N that was contaminated

6. Repeat step 2 to step 6 for all 15 locations listed on the NanoRacks Swab Kit label.

NOTE: An additional large Ziplock Bag is provided (stowed inside the same bag as the NanoRacks Swab Kits) to use per crew preference to separate the used NanoRacks Swab Kits from the clean (unused) NanoRacks Swab Kits for crew efficiency during sampling.

\section{ISS Crew}

Swabbing was conducted during Expedition 39

(http://www.nasa.gov/mission_pages/station/expeditions/expedition39/index.html). The crew included NASA astronauts Steve Swanson and Rick Mastracchio and Russian cosmonauts Oleg Artemyev, Alexander Skvortsov, and Mikhail Tyurin. Japan Aerospace Exploration Agency (JAXA) astronaut Koichi Wakata was the commander for this expedition, and is the astronaut who performed the swabbing.

\section{Sampling site choice}

These surfaces were chosen in an attempt to sample surfaces analogous to those sampled in the pilot study for the Wildlife of Our Homes project (Dunn et al. 2013). For this study, involving 40 homes, volunteers swabbed nine surfaces in their homes: kitchen cutting board, kitchen counter, a shelf inside a refrigerator, toilet seat, pillowcase, exterior handle of the main door into the house, television screen, the upper door trim on the outside surface of an exterior door, and the upper door trim on an interior door. We were not granted access to all corresponding surfaces aboard the ISS. The kitchen surfaces aboard the ISS are in the Russian module, which we did not have permission to access, swabbing the toilet seat was deemed inappropriate due to biosafety concerns, and the exterior surfaces are accessible only via an "Extra-vehicular Activity" (space walk), which was not requested for this experiment. We also sought to collect samples that would be analogous to the cell phone and shoe samples that were being obtained from thousands of Citizen Scientists across the country in a different component of Project MERCCURI. A final constraint was the limitation of only 15 swabs that was imposed by NASA, severely limiting the number of replicates we could collect. See Table 1 for a list of the ISS sampling sites and to which Earth samples they were intended to be analogous.

Upon successful completion of the swabbing on May 9, 2014, http://blogs.nasa.gov/stationreport/2014/05/09/iss-daily-summary-report-050914/, all swabs were stored at $-80^{\circ} \mathrm{C}$ in the Minus Eighty-degree Laboratory Freezer for ISS (MELFI) freezer onboard the ISS, until transfer to the SpaceX Dragon spacecraft. In the Dragon, the swabs were stored at $-80^{\circ} \mathrm{C}$ in the General Laboratory Active Cryogenic ISS Experiment Refrigerator (GLACIER), that runs off of Dragon's batteries until it is plugged in (either to the ISS or on the ground.) The Dragon re-entered the Earth's atmosphere and splashed 
180 down in the Pacific Ocean at 12:05 pm PT on May 18, 2014. Samples were transferred to a 181 cooler with dry ice, and shipped to the Earth Microbiome Project (EMP) lab

182 (http://earthmicrobiome.org)(Gilbert et al. 2011).

\section{DNA Extraction and Library Preparation}

184 All samples were prepared using a modified version of the Mo BIO UltraClean®-htp 96 185 Well Swab DNA Kit (MO BIO). Samples were purified using the Zymo ZR-96 DNA Cleanup 186 and Concentrator ${ }^{\mathrm{TM}}-5$ kit according to Zymo Protocol (Zymo). DNA was then amplified 187 using the EMP barcoded primer set, adapted for the Illumina HiSeq2000 and MiSeq by 188 adding nine extra bases in the adapter region of the forward amplification primer that 189 support paired-end sequencing. The V4 region of the 16S rRNA gene (515F-806R) was

190

191

192

193

194

195 amplified with region-specific primers that included the Illumina flowcell adapter sequences and a twelve-base barcode sequence. Each 25 ul PCR reaction contained the following: 12 ul of PCR water certified DNA-free (MO BIO), 10 ul of 1x 5 Prime HotMasterMix (5 Prime), 1 ul of Forward Primer ( 5 uM concentration, 200 pM final), 1 ul of Golay Barcode Tagged Reverse Primer (5 uM concentration, $200 \mathrm{pM}$ final), and $1 \mathrm{ul}$ of template DNA. The conditions for PCR were as follows: $94^{\circ} \mathrm{C}$ for 3 minutes to denature the DNA, with 35 cycles at $94{ }^{\circ} \mathrm{C}$ for $45 \mathrm{~s}, 50{ }^{\circ} \mathrm{C}$ for $60 \mathrm{~s}$, and $72{ }^{\circ} \mathrm{C}$ for $90 \mathrm{~s}$, with a final extension of $10 \mathrm{~min}$ at $72{ }^{\circ} \mathrm{C}$ to ensure complete amplification. Amplicons were quantified using PicoGreen (Invitrogen) and a plate reader. Once quantified, different volumes of each of the products were pooled into a single tube so that each amplicon was represented

200

201 equally. This pool was then cleaned up using UltraClean ${ }^{\circledR}$ PCR Clean-Up Kit (MO BIO), and then quantified using Qubit (Invitrogen). Sequencing of the prepared library was

202

203

204 performed on the Illumina MiSeq platform, using the sequencing primers and procedures described in the supplementary methods of (Caporaso 2012).

\section{Bioinformatic Analysis}

206

207

Unless otherwise noted, all microbial community analyses were conducted using the QIIME workflow version 1.8 or R (R-project 2014). All python scripts referred to are components of QIIME (Caporaso et al. 2010).

Demultiplex and QC: An in-house script was used to assign sequences to samples, using dual-index barcoding. This script is available on github (https://github.com/gjospin/Demul_trim_prep). This script allows for 1 base pair difference per barcode. The paired reads were then aligned and a consensus was computed using FLASH (Magoc and Salzberg 2011) with maximum overlap of 120 and a minimum overlap of 70 (other parameters were left as default). The custom script automatically demultiplexes the data into fastq files, executes FLASH, and parses its results to reformat the sequences with appropriate naming conventions for QIIME v. 1.8.0 in fasta format.

OTU assignment and QC: Chimeric sequences were identified using usearch61 as implemented in the identify_chimeric_seqs.py script, resulting in the removal of 8760 sequences. The pick_open_reference_otus.py script was used to cluster sequences at $97 \%$ similarity to generate OTUs (Operational Taxonomic Units, a proxy for species). Taxonomy 
221 gg_13_8_otus reference taxonomy provided by the Greengenes Database Consortium

222 (http://greengenes.secondgenome.com) (McDonald et al. 2011). OTUs that were classified

223 as chloroplasts or mitochondria were removed from further analysis. The number of high-

224 quality sequences remaining per sample ranged from 26831 to 77843 (see Table 1). All

225 subsequent beta diversity analyses (comparisons across samples) were performed with all

226 samples rarefied to 26830 sequences.

\section{Comparison of ISS surfaces to analogous surfaces in homes on Earth and to the}

228 Human Microbiome Project

229 The sequences and associated metadata from a 40-home pilot study for the Wildlife of Our

230 Homes Project are available for download from Figshare (Menninger; 2013). We also

231 obtained 100 samples from each of 13 body sites from the HMP Data Portal

232 (http://hmpdacc.org/HM16STR/)(Huttenhower et al. 2012)(Gevers et al. 2012). These two

233 additional datasets were used in a combined analysis with the ISS sequences presented

234 here. Because the sequences from the three projects are not all the same lengths, each

235 dataset was independently analyzed using a closed-reference OTU-picking approach, with a

$23697 \%$ similarity cutoff, and the resultant biom tables were merged with the

237 merge_otu_tables.py script. While the closed-reference approach will miss any novel taxa,

238 this was required since both of our comparison datasets were analyzed this way. To

239 account for uneven sampling depth, all samples in the combined analysis were rarefied to

2401000 sequences. Shannon diversity, as well as non-metric multidimensional scaling

241 (NMDS) based on Bray-Curtis (Bray and Curtis 1957)and Unweighted Unifrac (Lozupone

242 and Knight 2005) distances were computed and plotted using Phyloseq (McMurdie and

243 Holmes 2013) and the ggplot2 (Wilkinson 2011) packages in R (R-project 2014).

\section{Comparison to rooms with mechanical ventilation or open windows.}

245 We obtained a list of human pathogens, compiled by Kembel et al, 2012 from the author.

246 We then used BLAST (Altschul et al. 1990) to search a representative sequence from each

247 of the ISS OTUs against the NCBI Reference Sequence (RefSeq) database (Pruitt 2004).

248 OTUs with $97 \%$ similarity to an organism that was on the list of known pathogens were

249 flagged as "related to a known human pathogen". The phylogenetic diversity (Faith's PD)

250 was calculated using the alpha_diversity.py script, with samples rarefied to 700 sequences.

\section{Results and Discussion}

252 Overall taxonomic diversity of ISS surfaces and comparison to previous high-throughput 16S rDNA study

254

255

256

257

258

After filtering chimeric and eukaryotic sequences from the data, the number of sequences per surface sampled ranged from 26,221 - 76,656. Open-reference clustering at 97\% similarity resulted in 12,554 OTUs (OTU is a proxy for microbial "species".) This exceeds the number of species observed by Venkateswaran et al. 2014, and Ichijo et al. 2016 which is not surprising, given the increased sampling depth in this study $(\sim 1$ million versus $\sim$ 
$25950,000-71,000$ high-quality sequences.) Our study also had four notable, qualitative

260 differences from these earlier studies. In Venkateswaran et al. 2014, more than $90 \%$ of all

261 sequences were assigned to 4 bacterial genera (Corynebacterium, Propionibacterium,

262 Staphylococcus, and Streptococcus), while in the study here, they comprised only $24 \%$ of the

263 data (9.6\%, 0.05\%, 10.7\%, and 3.6\%, respectively). Ichijo et al. 2016 didn't report genus

264 level taxonomy but the phyla containing these groups (Firmicutes and Actinobacteria)

265 were highly abundant in all samples. Second, Venkateswaran et al. found no evidence of

266 archaea in their samples, even when interrogating with archaeal-specific primers, but we

267 did find evidence for a very low-abundance archaeal presence (2335 sequences, from three

268 archaeal phyla). No archaeal results were reported by Ichijo et al. 2016. Next, despite the

269 fact that Venkateswaran et al. were able to culture many spore-forming organisms from

270 their samples, they observed no sequence data from putative spore-forming organisms.

271 However, a large percentage of sequences in our study are from spore-forming genera:

$272 \quad 20.9 \%$ Bacillus and 9.6\% Clostridium. These differences are potentially due to differences in

273 PCR primers and/or DNA extraction method, both of which have known taxonomic biases

274 (Brooks et al. 2015). Lastly Ichijo et al. 2016 noted a significant amount of both

275 Legionellaceae and Neiseriaceae which are both of potential concerns as families

276 containing may pathogenic members. However, our study observed no OTUs for these

277 groups which is most likely due to sampling site differences or PCR primer differences as

278 noted above.

279 The 19 most abundant orders found in our study represent $93.8 \%$ of the data (Figure 1).

280 Within each of these 19 orders, the most abundant genus found in our samples tends to be

281 human-associated (Table 2). This is not surprising, as the only source of microbial influx is

282 via occasional crew and cargo deliveries aboard spacecraft that have been stringently

283 cleaned to avoid microbial contamination. It should be noted, as with all $16 \mathrm{~S}$ rDNA gene

284 surveys, that nothing can be said about the viability of these bacteria. Typically much of the

285 bacterial DNA on a surface is from dead or non-viable organisms. In built environments on

286 earth this DNA is assumed to come from many sources including outdoor air, soil, and the

287 passage of people and animals. On the ISS all of these taxa, viable or not, represent

288 organisms that have managed to survive the various protocols designed to limit them, the

289 most likely passage being on the crew themselves.

290 There were no apparent biogeographical patterns on the ISS surfaces. That is, there were

291 no significant differences between samples obtained from the different modules (crew vs

292 lab) or different surface types (keyboards, vents, or handheld mics). This can be visualized

293 in Figure 2, in which each point represents one of the 15 samples, and the distance between

294 samples indicates the overall difference in community composition. In Panel A, the metric

295 used to calculate the distance between samples is the Bray-Curtis dissimilarity, and in

296 Panel B, an alternative distance metric (Unifrac) is used, which takes into account the

297 phylogenetic distance between the OTUs in samples. For the most part, all 15 samples form

298 a tight cluster on the NMDS plots, but there is one sample, the starboard crew vent, that

299 appears distinct from all of the other samples in Panel A. In Panel B, that same sample, as

300 well as the aft lab vent sample appear separate from the others. In order to visualize which

301 OTUs are contributing the most to the uniqueness of those samples, we looked at the

302 overall distribution of the most abundant bacterial families in those samples. The three 
303 most abundant families in the starboard crew vent sample are Bacteroidaceae,

304 Ruminococcaceae, and Verrumicrobiaceae (comprising $60.1 \%$ of all sequences); and the

305 three most abundant families in the aft lab vent sample are Rikenellaceae, Bacteroidales

306 S24-7, and Lactobacillaceae (comprising 60\% of all sequences). In Figure 3, the relative

307 abundance of these six families in all 15 samples from the ISS provides a clear indication

308 that they are driving the distinctiveness of those two samples.

309 The massive increase in environmental 16S rDNA gene surveys over the last several years

310 has seen a greater understanding of the caveats and limitations with this kind of data, in

311 parallel with their unambiguous utility in understanding microbial communities. When this

312 experiment was designed in 2012, negative kit controls were not common but now they are

313 considered standard for good reason (Salter et al. 2014). Lacking a kit control, we cannot

314 say for certain which low-level taxa may have come from the swabs or reagents used

315 themselves.

\section{6}

Comparison to the microbial communities of homes on Earth and from the Human Microbiome Project

To put the microbial communities that we found on ISS surfaces in the context of homes on Earth, we compared them to the communities found by citizen scientists when they swabbed nine surfaces throughout 40 homes, as part of the "Wildlife of Our Homes" project (Dunn et al. 2013). We found that the ISS and homes on Earth were significantly different from each other, both based on the Bray-Curtis dissimilarity (adonis, $\mathrm{R}^{2}=0.0666, \mathrm{P}=0.001$ ) and the Unifrac distance (adonis, $\mathrm{R}^{2}=0.04189, \mathrm{P}=0.001$ ). These differences can be visualized in the ordination plots in Figure $4 \mathrm{~A}$ and B.

325 It is perhaps not surprising that the insular environment of the ISS would be unlike homes on Earth. Unlike the ISS, homes on Earth are exposed to a variety of sources of microbes, including the outdoor air, tracked-in soil, plants, pets, and human inhabitants (Barberán et al. 2015) (Barberán et al. 2015). The dominant source of microbes on the ISS is presumably the human microbiome. All spacecraft and cargo undergo rigorous decontamination procedures before launch to rendezvous with the ISS. Therefore, we hypothesized that the microbial communities of the ISS surfaces might be more similar to human-associated microbial communities than Earth home surfaces. To test this hypothesis, we obtained $16 \mathrm{~S}$ rDNA sequence data for 100 random samples from each of 13 body sites from the HMP Data Portal (http://hmpdacc.org/HM16STR/)(Huttenhower et al. 2012) (Gevers et al. 2012). The microbial communities associated with the ISS, homes on Earth, and the HMP samples were significantly different from each other (adonis, $\mathrm{R}^{2}=0.08, \mathrm{P}<0.001$ ) (Also see Figure 5). We note that as with any meta-analysis, this difference could be also be partly due to differences in sample collection/preparation. However, the ISS communities are significantly more similar to the Earth home samples than the HMP samples (Student's ttest, $\mathrm{p}<0.00001$ ). This combined analysis also indicates that the starboard crew vent sample, which appears quite distinct from the rest of the ISS samples in Figure 2A, is more similar to the human gastrointestinal HMP samples, which is corroborated by the dominance of animal gut-related OTUs found in that sample (see Figure 3, and Table 2.) 
344 Finally, because the ISS is designed only to house six crew members, for a stay of six 345 months each, only 220 individuals have visited the ISS since the year 2000 . We

346 hypothesized that there might be a relatively low microbial diversity on the ISS, either due 347 to having a few total number of species, or due to the dominance of a very few species. In

348 Figure 6, we note that Shannon diversity (which takes into account both the number of 349 species present, and how evenly our sequences are distributed throughout those species) is 350 actually relatively high on the ISS.

Comparison to rooms with mechanical ventilation or open windows.

352

353

354

355

356

357

358

359

360

361

362

363

364

365

366

367
Kembel et al., 2012, showed that rooms in a health-care facility that were primarily ventilated via an open window had greater phylogenetic diversity and lower proportion of OTUs closely related to known human pathogens than rooms that were mechanically ventilated. The only window on the ISS is never opened, and the doors are opened only briefly, every few months. Therefore, we hypothesized that for the samples from the ISS, the phylogenetic diversity would be lower and the proportion of OTUs closely related to known human pathogens would be higher than that seen for mechanically ventilated rooms. To test this hypothesis, we obtained the list of known human pathogens compiled by Kembel et al., 2012, and followed their procedure to identify the proportion of OTUs in the ISS samples that were closely related to them (see Methods for details). Surprisingly, but reassuringly, we found that the ISS samples are similar in both phylogenetic diversity and the proportion of OTUs closely related to known human pathogens as compared to the mechanically ventilated rooms in the health-care facility (Figure 7). As with the studies above, some observed variance may be due to differences in sample collection/preparation.

\section{Conclusion}

This is the first time that the ISS has been analyzed in the broader context of the "microbiology of the built environment", and is the most in-depth comparison of the microbial communities found on the ISS to those found either in buildings or in the human microbiome. Perhaps surprisingly, given the extreme rarity of exchange with any external microbes, we found the ISS to be species-rich, and more similar to the surfaces of human homes on Earth than it is to human bodies. We found that the ISS is home to at least 12,554 distinct microbial species, including Archea in very low abundance, and that the proportion of species that are closely related to known human pathogens is on par with similar built environments on Earth. Given the low number of samples in this study, no viability assessment, as well as the lack of sample preparation control we view these results as simply a starting place for more detailed future studies.

As outlined in the 2010 U.S. National Space Policy and in the bipartisan NASA Authorization Act of 2010, NASA is targeting the 2030s for a manned spaceflight to Mars, with one ultimate goal of having people live and work on the Martian surface (see www.nasa.gov/exploration and www.nasa.gov/mars). We know that the microbial 
384

385

386

387

388

389

390

391

392

393

394

395

396

397

398

399

400

401

402

403

404

405

406

407

408

409

410

411

412

413

414

415

416

417

communities found in our terrestrial built environments play an important role in human health. Therefore it's crucial to characterize and understand the microbial population of the only environment in which people are currently living and working in space. This study is one small step in that direction.

\section{Data Accessions}

Sequencing data has been deposited at NCBI under BioProject PRJNA376404. All data and analysis files are available on FigShare https://doi.org/10.6084/m9.figshare.4244123.v3

\section{Acknowledgements}

The authors would like to thank Summer Williams for the inception of the idea to get Science Cheerleader involved in space research. Also thanks to Carl Carruthers at Nanoracks LLC for managing our space payload. We are also grateful to Holly Menninger and Rob Dunn for sharing data from the Wildlife of Our Homes pilot project, and Steve Kembel for publishing the original version of Figure 7 in an open-access journal, as well as sharing the underlying data.

\section{References}

Altschul, SF, W Gish, W Miller, EW Myers, and DJ Lipman. 1990. "Basic Local Alignment Search Tool.." J Mol Biol 215: 403-10.

Albert Barberán, Robert R. Dunn, Brian J. Reich, Krishna Pacifici, Eric B. Laber, Holly L. Menninger, James M. Morton, Jessica B. Henley, Jonathan W. Leff, Shelly L. Miller, Noah Fierer. 2015. "The Ecology of Microscopic Life in Household Dust." Proceedings of the Royal Society B: Biological Sciences 282 (1814). The Royal Society: 20151139. doi:10.1098/rspb.2015.1139.

Barberán, Albert, Joshua Ladau, Jonathan W. Leff, Katherine S. Pollard, Holly L. Menninger, Robert R. Dunn, and Noah Fierer. 2015. "Continental-Scale Distributions of Dust-Associated Bacteria and Fungi." Proceedings of the National Academy of Sciences 112 (18). Proceedings of the National Academy of Sciences: 5756-61. doi:10.1073/pnas.1420815112.

Bray, J. Roger, and J. T. Curtis. 1957. "An Ordination of the Upland Forest Communities of Southern Wisconsin." Ecological Monographs 27 (4). JSTOR: 325. doi:10.2307/1942268.

Brooks JP, Edwards DJ, Harwich MD Jr, Rivera MC, Fettweis JM, Serrano MG, Reris RA, Sheth NU, Huang B, Girerd P; Vaginal Microbiome Consortium, Strauss JF 3rd, Jefferson KK, Buck GA. 2015. "The Truth about Metagenomics: Quantifying and Counteracting Bias in 16S 
418 RRNA Studies." BMC Microbiology 15 (1). Springer Science \$ $\backslash$ mathplus\$ Business Media. 419 doi:10.1186/s12866-015-0351-6.

420 Caporaso, Greg. 2012. "Ultra High-Throughput Microbial Ecology: Software Sequencing and 421 Practice for Studying Tens of Thousands of Environments." SciVee Inc.

422 doi:10.4016/46410.01.

423 J Gregory Caporaso , Justin Kuczynski , Jesse Stombaugh , Kyle Bittinger , Frederic D

424 Bushman , Elizabeth K Costello , Noah Fierer , Antonio Gonzalez Peña , Julia K Goodrich , 425 Jeffrey I Gordon, Gavin A Huttley, Scott T Kelley, Dan Knights, Jeremy E Koenig, Ruth E 426 Ley, Catherine A Lozupone, Daniel McDonald, Brian D Muegge, Meg Pirrung, Jens Reeder 427

428

429

430

431

432

433

434

435

436

437

438

439

440

441

442

443

444

445

446

447

448

449

450

451

452

453

454

455

456

457

458 Yatsunenko, Jesse Zaneveld and Rob Knight. 2010. "OIIME Allows Analysis of HighThroughput Community Sequencing Data." Nat Meth 7 (5). Nature Publishing Group: 33536. doi:10.1038/nmeth.f.303.

Castro, VA, AN Thrasher, M Healy, CM Ott, and DL Pierson. 2004. "Microbial Characterization during the Early Habitation of the International Space Station.." Microb Ecol 47: 119-26.

Dunn, RR, N Fierer, JB Henley, JW Leff, and HL Menninger. 2013. "Home Life: Factors Structuring the Bacterial Diversity Found within and between Homes.." PLoS One 8: e64133.

Gevers, Dirk, Rob Knight, Joseph F. Petrosino, Katherine Huang, Amy L. McGuire, Bruce W. Birren, Karen E. Nelson, Owen White, Barbara A. Methé, and Curtis Huttenhower. 2012. "The Human Microbiome Project: A Community Resource for the Healthy Human Microbiome." PLoS Biology 10 (8). Public Library of Science (PLoS): e1001377. doi:10.1371/journal.pbio.1001377.

Jack A. Gilbert, Mark Bailey, Dawn Field, Noah Fierer, Jed A. Fuhrman, Bin Hu, Janet Jansson, Rob Knight, George A. Kowalchuk, Nikos C. Kyrpides, Folker Meyer, and Rick Stevens. 2011. "The Earth Microbiome Project: The Meeting Report for the 1st International Earth Microbiome Project Conference Shenzhen, China, June 13th-15th 2011." Standards in Genomic Sciences 5 (2). Springer Science \$ $\backslash$ mathplus\$ Business Media: 243-47. doi:10.4056/sigs.2134923.

Huttenhower C, Gevers D, Knight R, Abubucker S, Badger JH, Chinwalla AT, Creasy HH, Earl AM, FitzGerald MG, Fulton RS, Giglio MG, Hallsworth-Pepin K, Lobos EA, Madupu R, Magrini V, Martin JC, Mitreva M, Muzny DM, Sodergren EJ, Versalovic J, Wollam AM, Worley KC, Wortman JR, Young SK, Zeng Q, Aagaard KM, Abolude OO, Allen-Vercoe E, Alm EJ, Alvarado L, Andersen GL, Anderson S, Appelbaum E, Arachchi HM, Armitage G, Arze CA, Ayvaz T, Baker CC, Begg L, Belachew T, Bhonagiri V, Bihan M, Blaser MJ, Bloom T, Bonazzi V, Brooks J, Buck GA, Buhay CJ, Busam DA, Campbell JL, Canon SR, Cantarel BL, Chain PS, Chen IM, Chen L, Chhibba S, Chu K, Ciulla DM, Clemente JC, Clifton SW, Conlan S, Crabtree J, Cutting MA, Davidovics NJ, Davis CC, DeSantis TZ, Deal C, Delehaunty KD, Dewhirst FE, Deych E, Ding Y, Dooling DJ, Dugan SP, Dunne WM, Durkin A, Edgar RC, Erlich RL, Farmer CN, Farrell RM, Faust K, Feldgarden M, Felix VM, Fisher S, Fodor AA, Forney LJ, Foster L, Di Francesco 
459 V, Friedman J, Friedrich DC, Fronick CC, Fulton LL, Gao H, Garcia N, Giannoukos G, Giblin C, 460 Giovanni MY, Goldberg JM, Goll J, Gonzalez A, Griggs A, Gujja S, Haake SK, Haas BJ, Hamilton 461 HA, Harris EL, Hepburn TA, Herter B, Hoffmann DE, Holder ME, Howarth C, Huang KH, Huse 462 SM, Izard J, Jansson JK, Jiang H, Jordan C, Joshi V, Katancik JA, Keitel WA, Kelley ST, Kells C, 463 King NB, Knights D, Kong HH, Koren O, Koren S, Kota KC, Kovar CL, Kyrpides NC, La Rosa 464 PS, Lee SL, Lemon KP, Lennon N, Lewis CM, Lewis L, Ley RE, Li K, Liolios K, Liu B, Liu Y, Lo 465 CC, Lozupone CA, Lunsford R, Madden T, Mahurkar AA, Mannon PJ, Mardis ER, Markowitz 466 VM, Mavromatis K, McCorrison JM, McDonald D, McEwen J, McGuire AL, McInnes P, Mehta 467 T, Mihindukulasuriya KA, Miller JR, Minx PJ, Newsham I, Nusbaum C, O'Laughlin M, Orvis J, 468 Pagani I, Palaniappan K, Patel SM, Pearson M, Peterson J, Podar M, Pohl C, Pollard KS, Pop M, Priest ME, Proctor LM, Qin X, Raes J, Ravel J, Reid JG, Rho M, Rhodes R, Riehle KP, Rivera MC, Rodriguez-Mueller B, Rogers YH, Ross MC, Russ C, Sanka RK, Sankar P, Sathirapongsasuti J, Schloss JA, Schloss PD, Schmidt TM, Scholz M, Schriml L, Schubert AM, Segata N, Segre JA, Shannon WD, Sharp RR, Sharpton TJ, Shenoy N, Sheth NU, Simone GA, Singh I, Smillie CS, Sobel JD, Sommer DD, Spicer P, Sutton GG, Sykes SM, Tabbaa DG, Thiagarajan M, Tomlinson CM, Torralba M, Treangen TJ, Truty RM, Vishnivetskaya TA, Walker J, Wang L, Wang Z, Ward DV, Warren W, Watson MA, Wellington C, Wetterstrand KA, White JR, Wilczek-Boney K, Wu Y, Wylie KM, Wylie T, Yandava C, Ye L, Ye Y, Yooseph S, Youmans BP, Zhang L, Zhou Y, Zhu Y, Zoloth L, Zucker JD, Birren BW, Gibbs RA, Highlander SK, Methé BA, Nelson KE, Petrosino JF, Weinstock GM, Wilson RK, White 0. 2012. "Structure Function and Diversity of the Healthy Human Microbiome." Nature 486 (7402). Nature Publishing Group: 207-14. doi:10.1038/nature11234.

481 Ichijo, Tomoaki, Nobuyasu Yamaguchi, Fumiaki Tanigaki, Masaki Shirakawa, and Masao Nasu. 2016. "Four-Year Bacterial Monitoring in the International Space StationJapanese Experiment Module Kibo with Culture-Independent Approach." Npj Microgravity 2 (April). Nature Publishing Group: 16007. doi:10.1038/npjmgrav.2016.7.

485

486

487

488 489 490

491 492

493

494 495

496

497 498

499 500
Lozupone, C., and R. Knight. 2005. "UniFrac: a New Phylogenetic Method for Comparing Microbial Communities." Applied and Environmental Microbiology 71 (12). American Society for Microbiology: 8228-35. doi:10.1128/aem.71.12.8228-8235.2005.

Maule, J, N Wainwright, A Steele, L Monaco, H Morris, D Gunter, M Damon, and M Wells. 2009. "Rapid Culture-Independent Microbial Analysis Aboard the International Space Station (ISS).." Astrobiology 9: 759-75.

Magoc, T., and S. L. Salzberg. 2011. "FLASH: Fast Length Adjustment of Short Reads to Improve Genome Assemblies." Bioinformatics 27 (21). Oxford University Press (OUP): 2957-63. doi:10.1093/bioinformatics/btr507.

McDonald, Daniel, Morgan N Price, Julia Goodrich, Eric P Nawrocki, Todd Z DeSantis, Alexander Probst, Gary L Andersen, Rob Knight, and Philip Hugenholtz. 2011. “An Improved Greengenes Taxonomy with Explicit Ranks for Ecological and Evolutionary Analyses of Bacteria and Archaea." The ISME Journal 6 (3). Nature Publishing Group: 61018. doi:10.1038/ismej.2011.139.

McMurdie, Paul J., and Susan Holmes. 2013. "Phyloseq: An R Package for Reproducible Interactive Analysis and Graphics of Microbiome Census Data." Edited by Michael Watson. 
501 PLoS ONE 8 (4). Public Library of Science (PLoS): e61217.

502 doi:10.1371/journal.pone.0061217.

503 Menninger;, Robert R. Dunn; Noah Fierer; Jessica B. Henley; Jonathan Leff; Holly L. 2013. 504 "Home Life: Factors Structuring the Bacterial Diversity Found within and between Homes 505 (16S RRNA Sequence Data and Metadata)." Figshare. doi:10.6084/m9.figshare.674588.

506 Novikova, N, Boever P De, S Poddubko, E Deshevaya, N Polikarpov, N Rakova, I Coninx, and 507 M Mergeay. 2006. "Survey of Environmental Biocontamination on Board the International 508 Space Station.." Res Microbiol 157: 5-12.

509 Ott, CM, RJ Bruce, and DL Pierson. 2004. "Microbial Characterization of Free Floating 510 Condensate Aboard the Mir Space Station.." Microb Ecol 47: 133-36.

511 Pierson, Duane L. 2007. "Microbial Contamination of Spacecraft." Gravitational and Space 512 Research 14 (2).

513 Pruitt, K. D. 2004. "NCBI Reference Sequence (RefSeq): a Curated Non-Redundant Sequence 514 Database of Genomes Transcripts and Proteins." Nucleic Acids Research 33 (Database 515 issue). Oxford University Press (OUP): D501-D504. doi:10.1093/nar/gki025.

516 R-project. 2014. "R: A Language and Environment for Statistical Computing." Vienna, 517 Austria: R Foundation for Statistical Computing. http://www.R-project.org/.

518 Roberts, MS, JL Garland, and AL Mills. 2004. "Microbial Astronauts: Assembling Microbial 519 Communities for Advanced Life Support Systems.." Microb Ecol 47: 137-49.

520 Salter, SJ, MJ Cox, EM Turek, ST Calus, WO Cookson, MF Moffatt, P Turner, J Parkhill, NJ 521 Loman, and AW Walker. 2014. "Reagent and Laboratory Contamination Can Critically 522 Impact Sequence-Based Microbiome Analyses..” BMC Biol 12: 87.

523 Trexler, PC. 1964. "Gnotobiotic Techniques and Their Application to Spacecraft

524 Fabrication..” Life Sci Space Res 2: 433-39.

525 Venkateswaran, K, P Vaishampayan, J Cisneros, DL Pierson, SO Rogers, and J Perry. 2014. 526 "International Space Station Environmental Microbiome - Microbial Inventories of ISS 527 Filter Debris.." Appl Microbiol Biotechnol 98: 6453-66.

528 Wilkinson, Leland. 2011. "ggplot2: Elegant Graphics for Data Analysis by WICKHAM H.." 529 Biometrics 67 (2). Wiley-Blackwell: 678-79. doi:10.1111/j.1541-0420.2011.01616.x. 


\section{Figure 1}

Relative abundances of the most common bacterial families found on surfaces of the ISS.

Pie chart showing the relative abundances of the most common bacterial families found on the 15 surfaces of the International Space Station. This graph was produced using METAGENassist [26].

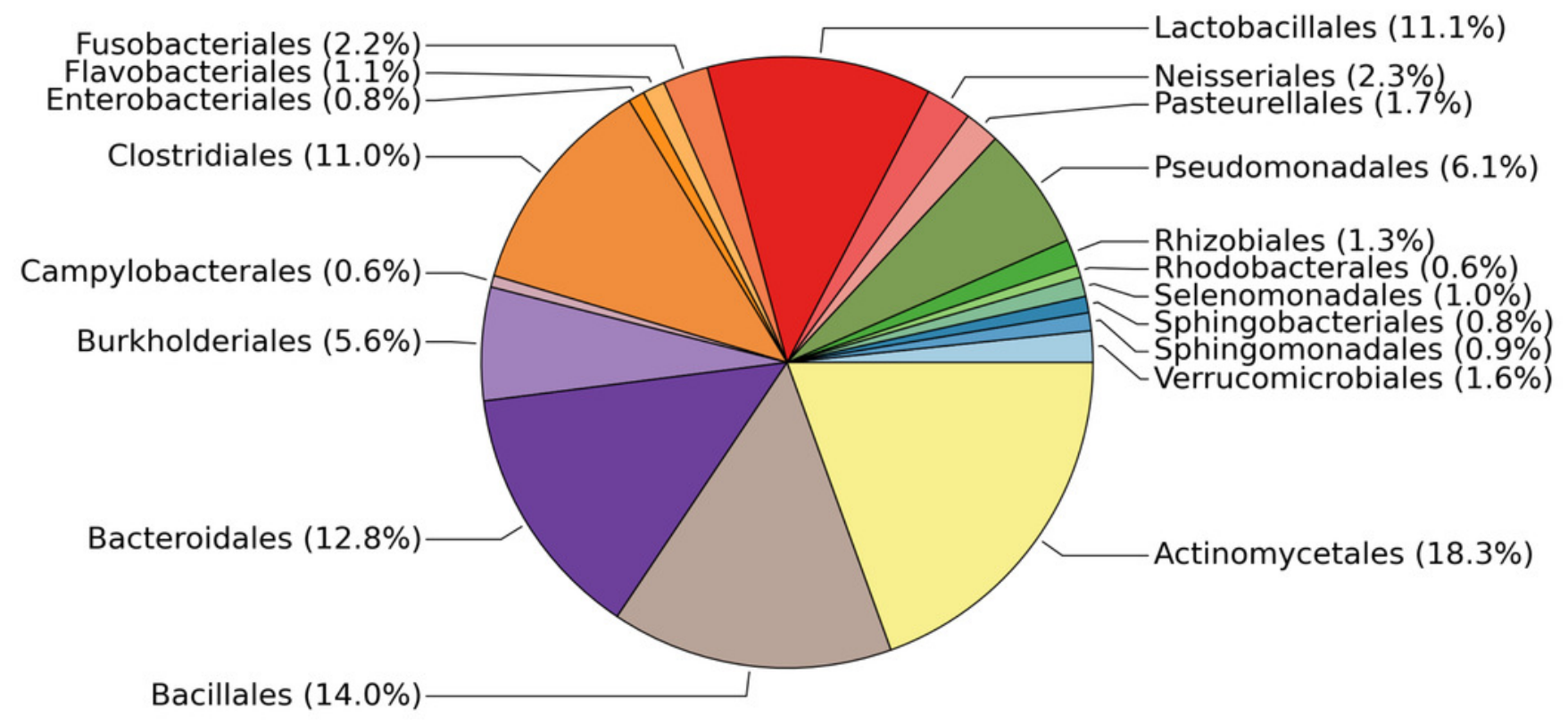




\section{Figure 2 (on next page)}

Non-metric multidimensional scaling (NMDS) ordination plots of 15 ISS surface samples Non-metric multidimensional scaling (NMDS) ordination plots, based on Bray-Curtis (Panel A) or Unweighted Unifrac (Panel B) distances between the samples obtained from the International Space Station. In these plots, points that are closer together have more similar microbial communities. Note, there is a (starboard) crew vent sample that does not cluster with the other ISS samples in Panel A, and in Panel B, a second sample (aft lab vent) appears closer to it. This graph was produced using the Phyloseq package [18] in R [13]. 


\section{Figure 3 (on next page)}

Most abundant bacterial families found in each of the two "outlier" samples on the ISS.

Bar chart showing the distribution across all samples of the 3 most abun- dant bacterial families found in each of the two samples (starboard crew vent and aft lab vent) that do not cluster with the others in Figure 2. All six of these families are known to be found in association with human (or animal) gastrointestinal tract. 


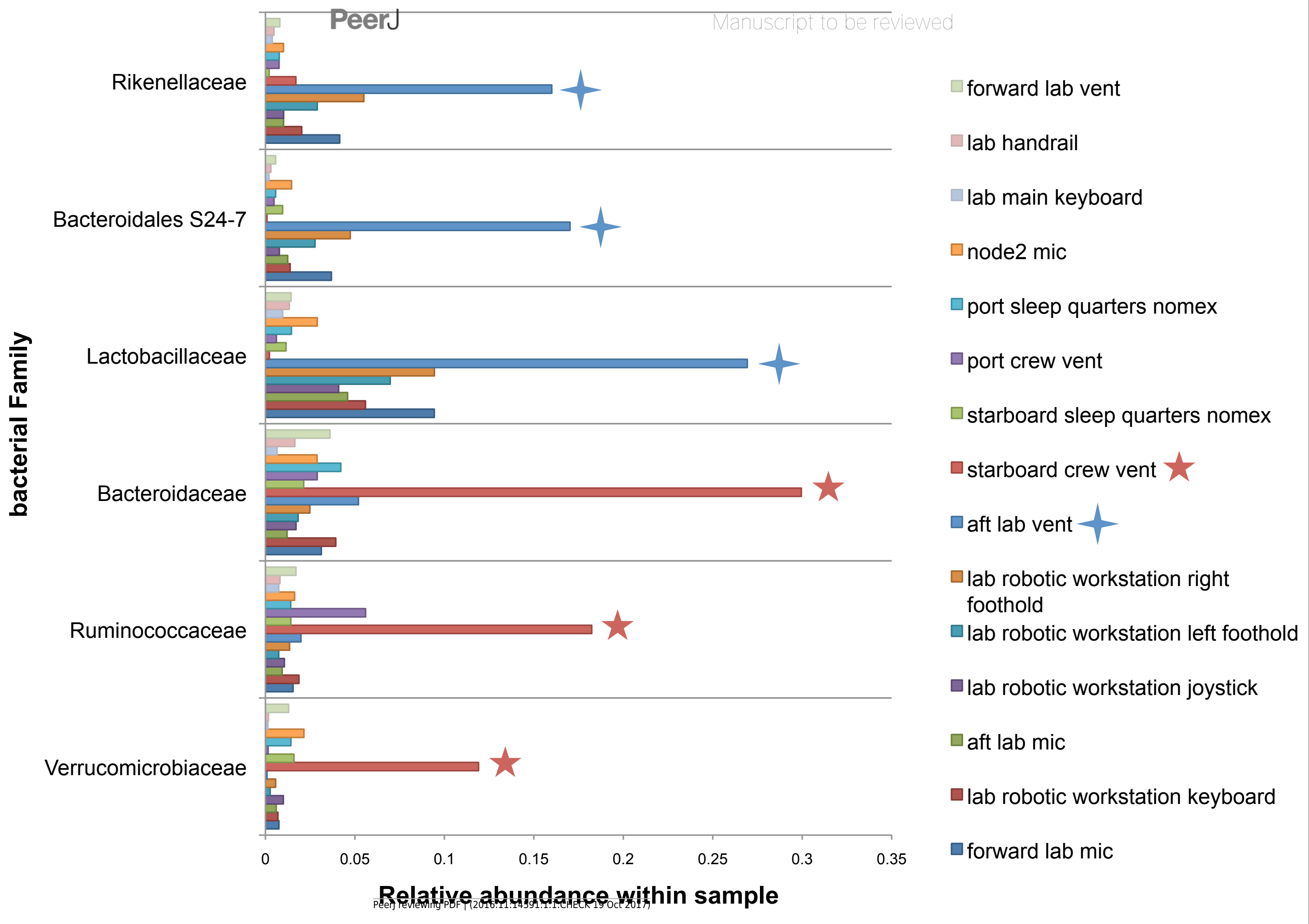




\section{Figure 4 (on next page)}

Non-metric multidimensional scaling (NMDS) ordination plots of ISS surface samples.

Non-metric multidimensional scaling (NMDS) ordination plots, based on Bray-Curtis (Panel A) or Unweighted Unifrac (Panel B) distances between samples obtained from the International Space Station and samples obtained from homes on Earth. In these plots, points that are closer together have more similar microbial communities. We found that the ISS samples and Earth home samples were significantly different from each other, both based on the Bray-Curtis dissimilarity (adonis, $\mathrm{R}^{2}=0.0666, \mathrm{P}=0.001$ ) and the Unifrac distance (adonis, $\mathrm{R}^{2}=0.04189, \mathrm{P}=0.001$ ). Note, the crew and lab vent samples that are distinct from the other ISS samples Figure 2, do not cluster with any of the Earth home surfaces. This graph was produced using the Phyloseq package [18] in R [13]. 


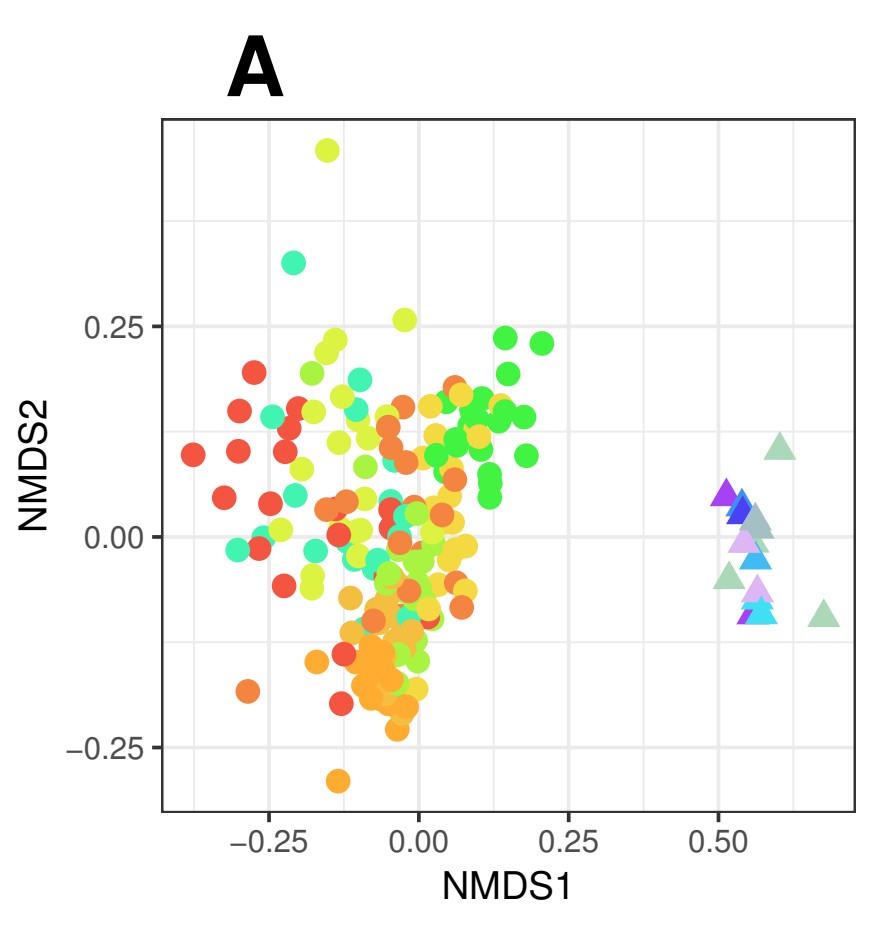

\section{Surface}

vent (ISS)

nomex (ISS)

mic (ISS)

- Node2 mic (ISS)

handrail (ISS)

foothold for RWS (ISS)

- joystick for RWS (ISS)

keyboard (ISS)

cutting board (Homes)

door handle (Homes)

exterior door trim (Homes)

interior door trim (Homes)

pillowcase (Homes)

refrigerator (Homes)

television (Homes)

toilet seat (Homes)

kitchen counter (Homes)

\section{Source}

- Homes

$\Delta$ ISS

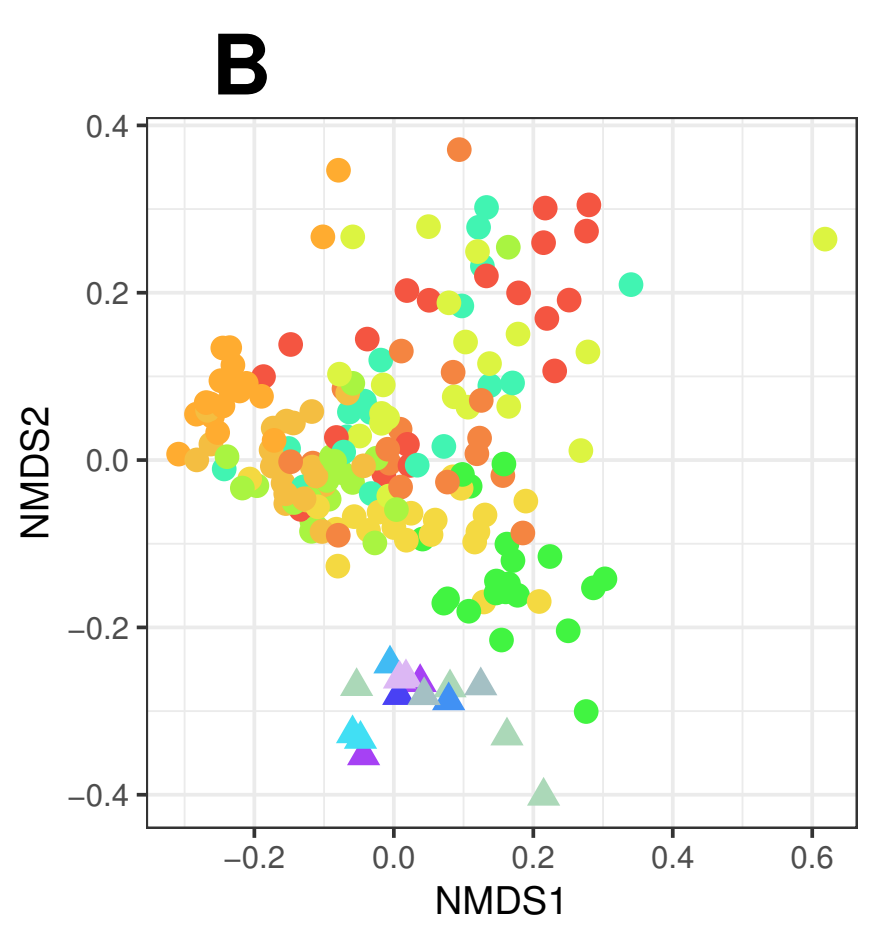

Surface

vent (ISS)

nomex (ISS)

mic (ISS)

Node2 mic (ISS)

handrail (ISS)

foothold for RWS (ISS)

joystick for RWS (ISS)

keyboard (ISS)

cutting board (Homes)

door handle (Homes)

exterior door trim (Homes)

interior door trim (Homes)

pillowcase (Homes)

refrigerator (Homes)

television (Homes)

toilet seat (Homes)

kitchen counter (Homes)

\section{Source}

- Homes

ISS 


\section{Figure 5}

NMDS plots showing clustering of ISS, Earth homes, and Human Microbiome Project body sites.

Non-metric multidimensional scaling (NMDS) ordination plots, based on Bray-Curtis (Panels A and C) or Unweighted Unifrac (Panels B and D) distances between samples obtained from the International Space Station, from homes on Earth, and from 13 body site from the Human Microbiome Project. The plots in panels A and B show identical data, as do the plots in panels $C$ and $D$. The points in A vs. B and C vs. D are colored differently as an aid for visualization. In these plots, points that are closer together have more similar microbial communities. The microbial communities associated with the ISS, homes on Earth, and the HMP samples were significantly different from each other (adonis, $R^{2}=0.08, P<0.001$ ). Note, the crew and lab vent samples that are distinct from the other ISS samples in Figure 2 are more similar to the human gastrointestinal tract samples from the HMP. This graph was produced using the Phyloseq package [18] in R [13]. 

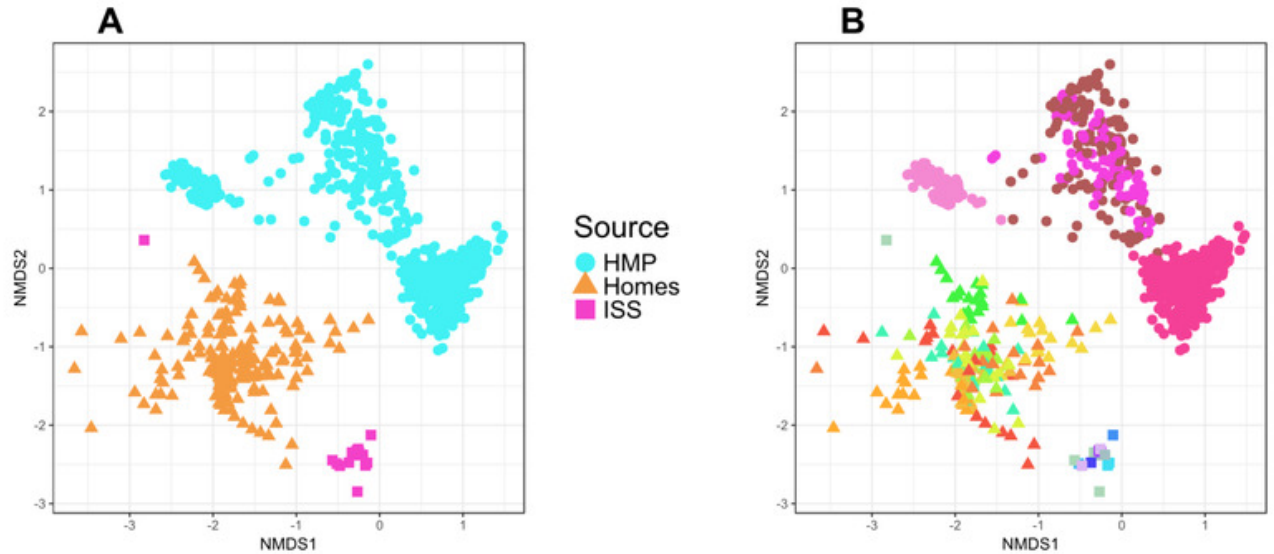

\section{Source \\ Q Homes}

HMP

a Homes
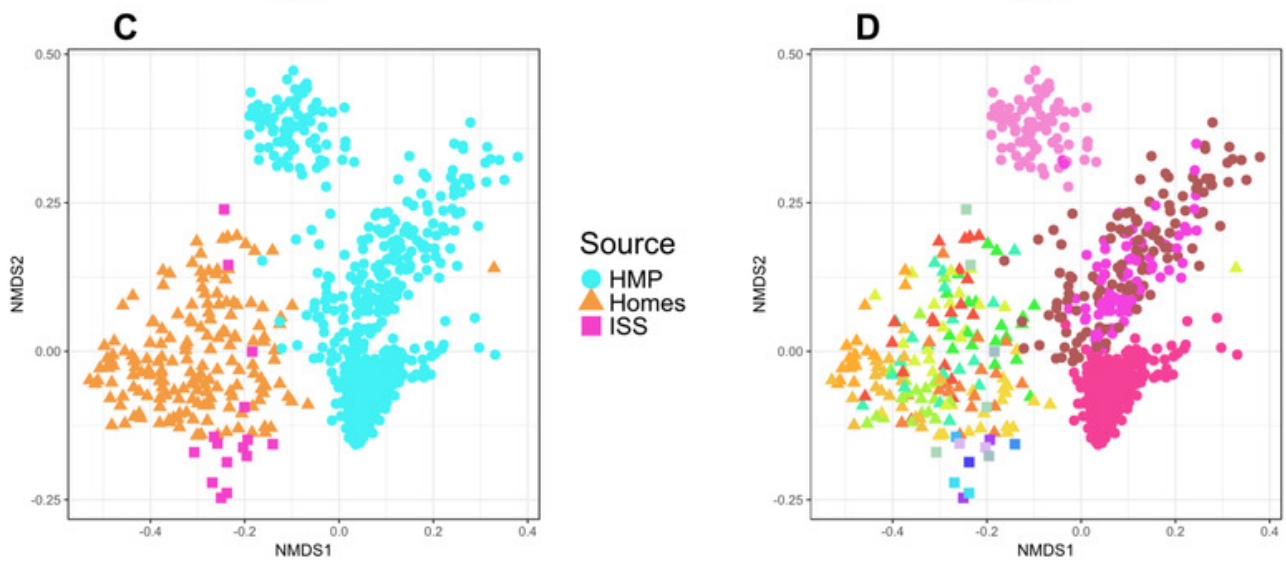

\section{Surface}

vent (ISS)

nomex (ISS)

mic (ISS)

Node2 mic (ISS)

handrail (ISS)

foothold for RWS (ISS)

joystick for RWS

keyboard (ISS

cutting board (Homes)

door handle (Homes)

exterior door trim (Homes)

interior door trim (Homes)

pillowcase (Homes)

refrigerator (Homes)

television (Homes)

television (Homes)
toilet seat (Homes)

kitchen counter (Homes)

gastrointestinal tract (HMP)

airways (HMP)

oral (HMP)

skin (HMP) 


\section{Figure 6 (on next page)}

Comparison of Shannon diversity among the ISS, Earth homes, and HMP body sites.

Shannon diversity, a measure of how many species there are as well as how evenly the counts of individuals are distributed across species is plotted for every sample. There is wide variation among the HMP samples, with the oral (blue) and gastrointestinal (green) samples typically having more diversity than the skin (pink) or airway (coral) samples. Surfaces on the International Space Station have relatively high Shannon diversity, on par with that of the most diverse HMP samples, and the average home sample. This graph was produced using the Phyloseq package [18] in R [13]. 


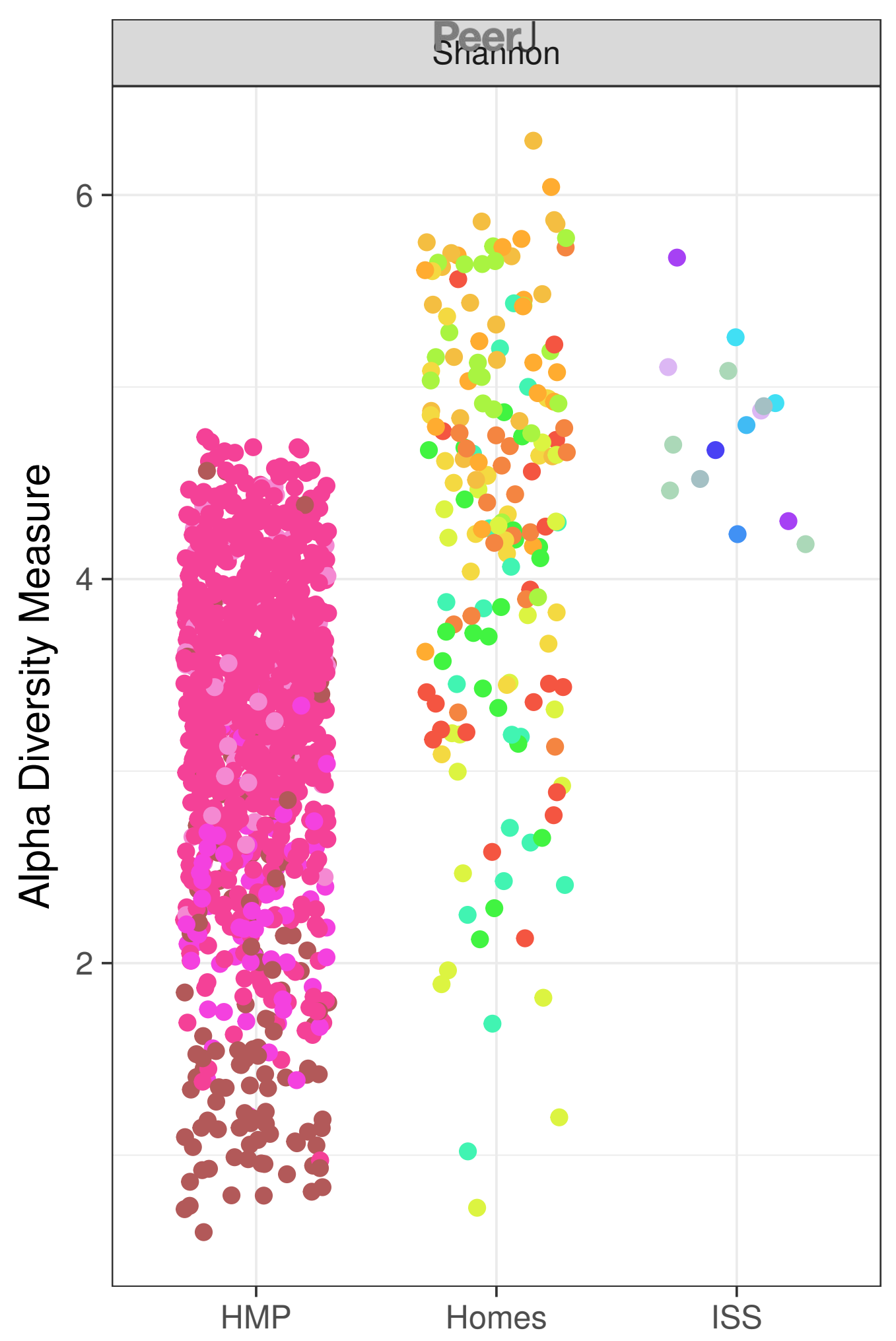

\section{Surface}

vent (ISS)

- nomex (ISS)

- mic (ISS)

- Node2 mic (ISS)

- handrail (ISS)

- foothold for RWS (ISS)

- joystick for RWS (ISS)

- keyboard (ISS)

- cutting board (Homes)

- door handle (Homes)

- exterior door trim (Homes) interior door trim (Homes) pillowcase (Homes) refrigerator (Homes) television (Homes)

- toilet seat (Homes)

- kitchen counter (Homes)

- gastrointestinal tract (HMP)

- airways (HMP)

- oral (HMP)

- skin (HMP)

SOUPCC ewing PDF | (2016:11:14591:1:1:CHECK 19 Oct 2017) 


\section{Figure 7}

Proportion of OTUs found in the ISS samples that were closely related (97\% sequence similarity) to human pathogens versus the phylogenetic diversity of those samples.

This figure was modified from Figure 4a. of [25]. The pink star represents the ISS samples. The plot shows the proportion of OTUs that were closely related (97\% sequence similarity) to human pathogens versus the phylogenetic diversity of those samples. 


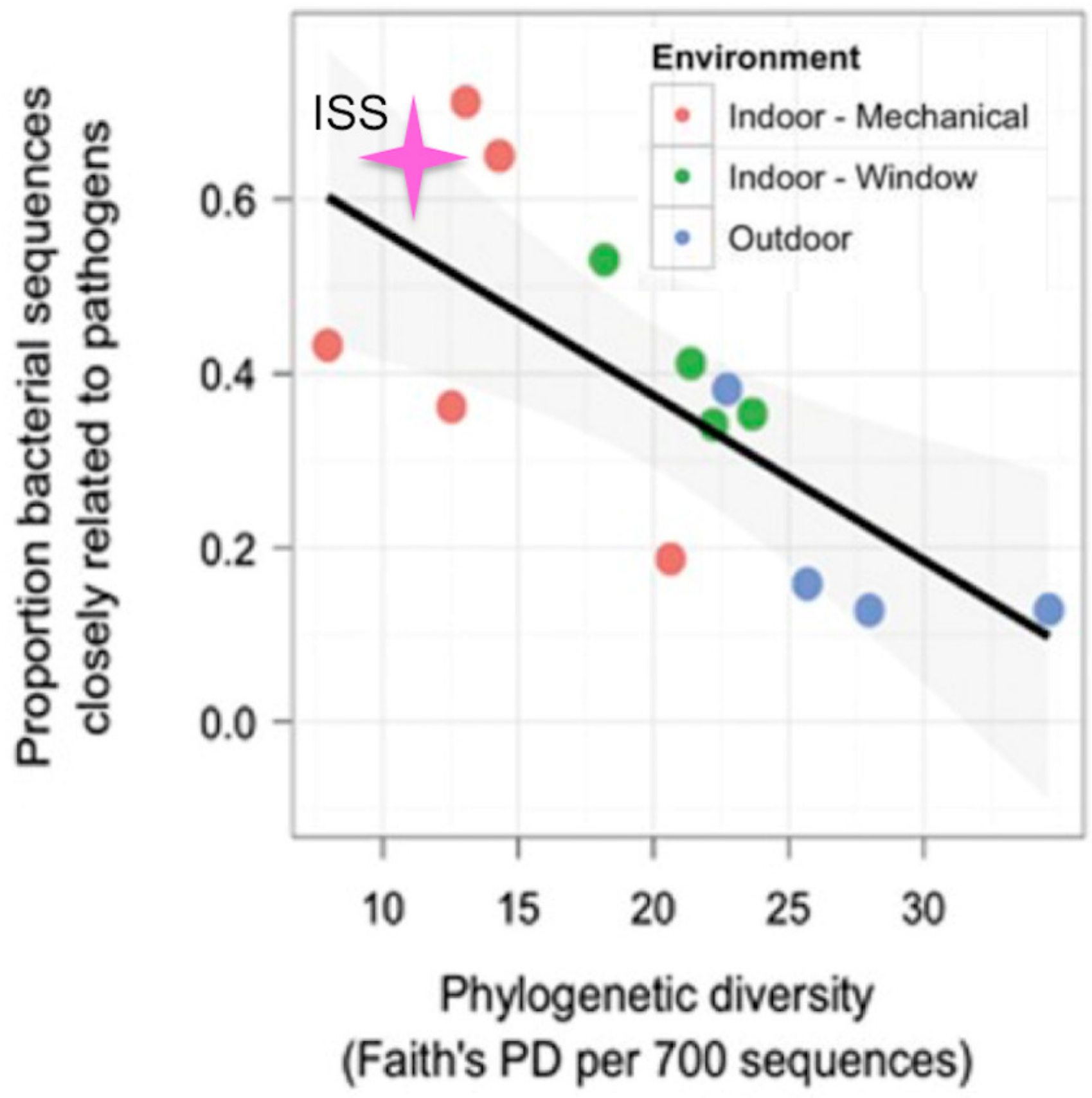




\section{Table 1 (on next page)}

ISS sample surface descriptions and sequence statistics. 
Table 1:

\section{Sample}

forward lab mic

lab robotic workstation

keyboard

aft lab mic

lab robotic workstation

joystick

lab robotic workstation left

foothold

lab robotic workstation right

foothold

aft lab vent

starboard crew vent

starboard sleep quarters

nomex

port crew vent

port sleep quarters nomex

node2 mic

lab main keyboard

lab handrail

forward lab vent

\section{Earth analog}

cell phone

none

cell phone

door handle

shoe

shoe

interior door trim

interior door trim

pillow

interior door trim

pillow

cell phone

none

door handle

interior door trim
Number of sequences obtained

45902

31612

63958

76198

77843

74023

64782

63280

26831

50418

61306

50416

62567

70418

57715
Number of Species Observed (OTUs at $\mathbf{9 7 \%}$ similarity)

1744

1320

2457

2820

1995

2129

1456

4294

1036

1757

1349

1429

1678

2904

2380 


\section{Table 2 (on next page)}

The most abundant organisms on the ISS are human-associated.

From each of the 19 orders shown in Figure 1, we selected the most abundant genus and conducted a literature review to identify whether or not it is known to occur in association with the human microbiome. 
Table 2:

Order

Actinomycetales
Bacillales
Bacteroidales
Lactobacillales
Clostridiales
Pseudomonadales
Burkholderiales

Neisseriales

Fusobacteriales

Pasteurellales

Verrucomicrobiales

Flavobacteriales

Selenomonadales

Sphingomonadales

Sphingobacteriales

Enterobacteriales

Rhizobiales

Campylobacterales

\section{$\%$ abundance}

dominant Genus

\subsection{Corynebacterium}

14 Staphylococcus unclassified Rikenellaceae/S24-

\subsection{7}

\subsection{Streptococcus}

11 Finegoldia

6.1 Pseudomonas unclassified

5.6 Comamonadaceae

\subsection{Neisseria}

2.2 Fusobacterium

\subsection{Haemophilus}

1.6 Akkermansia

1.1 Capnocytophaga

1 Selenomonas

0.9 Sphingomonas unclassified

0.8 Sphingobacteriales unclassified

\subsection{Enterobacteraceae}

0.6 Methylobacterium

0.6 Campylobacter common habitat

human skin, oral cavity human skin, oral cavity

animal gut human oral

cavity human skin human skin

environmental (Willems 2014)

human

mucous membranes human oral cavity human respiratory tract

(Murphy 2007)

human gut human oral cavity human oral cavity

(Liu 2015)

(Schwarzberg 2014)

(Belzer 2012)

(Zaura 2009)

(Ribeiro 2011)

environmental (Seifried 2015)

environmental (Steyn 1998)

animal gut (Linton 1988)

environmental (Knief 2010)

animal gut

(Young 2007) 